\title{
Effect of short-term orthokeratology lens or ordinary frame glasses wear on corneal thickness, corneal endothelial cells and vision correction in adolescents with low to moderate myopia
}

\author{
Shuyi Yuan, Shuxian Zhang, Yanglin Jiang and Lihua Li* (D)
}

\begin{abstract}
Background: We aimed to investigate the effect of short-term orthokeratology lens or frame glasses wear on corneal thickness, corneal endothelial cells and vision correction in adolescents with low to moderate myopia.

Methods: Data of 100 adolescents with low to moderate myopia were retrospectively analyzed. The patients were assigned into two groups. The experimental group were treated with night-wear orthokeratology lens, and control group were treated with ordinary frame glasses. Follow up was carried out at the $3 \mathrm{rd}$, 6th and 12th months of treatment. The naked-eye vision, diopter, corneal curvature, intraocular pressure, axial length, endothelial cell count and central corneal thickness were examined. Complications within 12 months were observed, and corneal fluorescein staining was graded.

Results: The naked-eye vision of the experimental group was significantly higher than that of the control group at the 3rd, 6th and 12th months, while the diopter of the experimental group was significantly lower than that of the control group at these time points. The corneal curvature of the experimental group was significantly decreased when comparing with that of the control group at the above 3 time points. The increase of axial length in the experimental group was significantly less than that in the control group at the 6th and 12th months.

Conclusions: Short-term orthokeratology lens wear can effectively improve the naked-eye vision in adolescents with low to moderate myopia without significant impact on the central corneal thickness and corneal endothelial cells. It is a relatively safe method to correct myopia.
\end{abstract}

Keywords: Orthokeratology lens, Frame glasses, Correction of myopia, Corneal thickness, Corneal endothelial cells

\section{Background}

Myopia refers to a pathological phenomenon that when parallel light passes through the dioptric system (the body is completely relaxed) and the focal point is located in front of the retina, so patients with myopia see distant blurred and near clear [1]. At present, although there are many studies on myopia, its pathogenesis is still unclear. Most studies indicate that myopia is related to genetic

\footnotetext{
* Correspondence: lilihua5802@163.com

Tianjin Eye Hospital, Tianjin Key Lab of Ophthalmology and Visual Science, Tianjin Eye Institute, No.4 Gansu Road, Heping District, Tianjin City 300022, China
}

(c) The Author(s). 2019 Open Access This article is distributed under the terms of the Creative Commons Attribution 4.0 International License (http://creativecommons.org/licenses/by/4.0/), which permits unrestricted use, distribution, and reproduction in any medium, provided you give appropriate credit to the original author(s) and the source, provide a link to the Creative Commons license, and indicate if changes were made. The Creative Commons Public Domain Dedication waiver (http://creativecommons.org/publicdomain/zero/1.0/) applies to the data made available in this article, unless otherwise stated.

and environmental factors [2]. Myopia, as a common disease among adolescents, has an increasing incidence and a trend toward the younger [3]. Statistics showed that the number of myopic patients in the world had reached 1.406 billion in 2000, and nearly 2 billion in 2010 , accounting for about $30 \%$ of the global population. It was predicted that the number of myopic patients worldwide might exceed 4.7 billion by 2050 , accounting for $50 \%$ of the global population [4]. Various long-term complications might occur in patients with the worsen of myopia, including cataract, glaucoma or even retinal detachment, which seriously impact the visual function 
Table 1 Grading of corneal fluorescein staining [13]

\begin{tabular}{lll}
\hline Grade & Degree of injury & Treatment \\
\hline 0 & A few dots of staining & No Treatment \\
I & $\begin{array}{l}\text { Increased spots showing a } \\
\text { scatter pattern }\end{array}$ & $\begin{array}{l}\text { Continuing lens wear and } \\
\text { using a small amount of eye } \\
\text { drop for cornea repair until the } \\
\text { obvious clinical manifestations, } \\
\text { disappearance of symptoms } \\
\text { dense and diffuse spots }\end{array}$ \\
III $\quad \begin{array}{ll}\text { A large number of stippling } \\
\text { stains, aggregation and fusion } \\
\text { occurred in a large range }\end{array}$ & $\begin{array}{l}\text { Stopping treatment, adjusting } \\
\text { the lens, and giving } \\
\text { symptomatic medication until } \\
\text { IV disappearance of }\end{array}$ \\
& $\begin{array}{l}\text { Diffuse stippling stains of the } \\
\text { whole cornea, mass fusion or } \\
\text { even complete epithelial loss }\end{array}$ & symptoms \\
\hline
\end{tabular}

of patients [5]. Research statistics showed that more than 300,000 people in China were blind due to myopia [6]. Therefore, the treatment for myopia is one of the major clinical issues.

The approaches to correct myopia in clinic include drug therapy, surgery, frame glasses and corneal contact lenses etc. [7]. The most common way of correction is using a pair of frame glasses for it is a simple method and the glasses can be changed at will according to the change of the patient's eyesight [8]. Drug treatment can be divided into long-term and short-term treatment. At present, low-concentration atropine has made favorable progress in controlling myopia, however, long-term use of drugs, which results side effects and drug resistance, needs to be further improved [9]. Surgical treatment is generally used in patients over 18 years and with stable diopters, which is not recommended for adolescents and children [10]. Corneal contact lens consists of a plurality of arc segments and includes three types: soft lens, rigid lens and orthokeratology (OK) lens. Among them, the OK lens plays a significant role against myopia and diopter change; besides, OK lenses only need to be worn at night, which has less influence on the wearer's daily

Table 2 Comparison of clinical data ( $\mathrm{n}, \%)$

\begin{tabular}{lllll}
\hline Factor & $\begin{array}{l}\text { Experimental group } \\
(n=53)\end{array}$ & $\begin{array}{l}\text { Control group } \\
(n=47)\end{array}$ & $x^{2}$ & $p$ \\
\hline $\begin{array}{l}\text { Gender } \\
\text { Male }\end{array}$ & $28(52.83)$ & $25(53.19)$ & & \\
$\quad$ Female & $25(47.17)$ & $22(46.81)$ & & \\
Age (year) & & & 0.045 & 0.832 \\
$>10$ years & $22(41.51)$ & $20(42.55)$ & & 0.916 \\
$\leq 10$ years & $31(58.49)$ & $27(57.45)$ & & \\
$\begin{array}{l}\text { Course of disease } \\
>6 \text { months }\end{array}$ & $10(18.87)$ & & 0.645 & 0.422 \\
$\leq 6$ months & $43(81.13)$ & $12(25.53)$ & & \\
Diopter & $3.84 \pm 0.92$ & $35(74.47)$ & & \\
\hline
\end{tabular}

Table 3 Comparison of naked-eye vision

\begin{tabular}{lllll}
\hline Group & $\begin{array}{l}\text { Control group } \\
(n=94)\end{array}$ & $\begin{array}{l}\text { Experimental group } \\
(n=106)\end{array}$ & $\mathrm{t}$ & $p$ \\
\hline Before treatment & $0.26 \pm 0.04$ & $0.25 \pm 0.05$ & 1.569 & 0.118 \\
The 3rd month & $0.25 \pm 0.05^{\mathrm{a}}$ & $0.56 \pm 0.15^{\mathrm{a}}$ & 20.063 & 0.000 \\
The 6th month & $0.23 \pm 0.05^{\mathrm{ab}}$ & $0.64 \pm 0.12^{\mathrm{ab}}$ & 32.176 & 0.000 \\
The 12th month & $0.20 \pm 0.04^{\mathrm{abc}}$ & $0.92 \pm 0.07^{\mathrm{abc}}$ & 90.548 & 0.000
\end{tabular}

Compared with before treatment, ${ }^{a} p<0.01$; compared with the $3 \mathrm{rd}$ month,

${ }^{\mathrm{b}} p<0.01$; compared with the 6th month, ${ }^{c} p<0.01$

work [11]. Some studies have shown that OK lens can effectively improve diopter of myopic patients, but the long-term directly contact of cornea gives constant mechanical pressure to the eyes, which results certain influence on the corneal layer of the patient [12]. Whether the short-term wear has influence on the endothelial cells and corneal thickness of the wearer is unclear.

Therefore, we carried out a study of short-term OK lens or frame glasses wear for myopia correction in juvenile patients with low to moderate myopia, so as to investigate the influence on central corneal thickness and corneal endothelial cells.

\section{Materials and methods}

\section{Clinical data}

The data of 100 adolescents (200 eyes) with low to moderate myopia admitted to Tianjin Eye Hospital from March 2016 to July 2017 were analyzed retrospectively and grouped according to the different correction methods. There were 53 patients in experimental group (28 males and 25 females, aged 8-15 years, with an average age of $11.4 \pm 2.2$ years) and 47 patients in control group ( 25 males and 22 females, aged $8-15$ years, with an average age of $11.0 \pm 2.3$ years). This study was approved by the Medical Ethics Committee of Tianjin Eye Hospital. Written informed consent form was obtained from all patients and their guardians.

\section{Inclusion criteria}

Patients were eligible if they were aged 8-15 years old; had diopter from $-1.50 \mathrm{D}$ to $-5.00 \mathrm{D}$, had with-the-rule astigmatism less than $1.50 \mathrm{D}$; had against-the-rule

Table 4 Comparison of diopter (D)

\begin{tabular}{lllll}
\hline Group & $\begin{array}{l}\text { Control group } \\
(n=94)\end{array}$ & $\begin{array}{l}\text { Experimental group } \\
(n=106)\end{array}$ & $\mathrm{t}$ & $p$ \\
\hline Before treatment & $3.84 \pm 0.92$ & $3.62 \pm 0.84$ & 1.758 & 0.080 \\
The 3rd month & $4.25 \pm 1.20^{\mathrm{a}}$ & $0.53 \pm 0.25^{\mathrm{a}}$ & 29.486 & 0.000 \\
The 6th month & $4.83 \pm 1.26^{\mathrm{ab}}$ & $0.44 \pm 0.19^{\mathrm{ab}}$ & 33.435 & 0.000 \\
The 12th month & $5.36 \pm 1.32^{\mathrm{abc}}$ & $0.29 \pm 0.08^{\mathrm{abc}}$ & 37.168 & 0.000
\end{tabular}

Compared with before treatment, ${ }^{a} p<0.01$; compared with the 3rd month, ${ }^{\mathrm{b}} p<0.01$; compared with the 6th month, ${ }^{c} p<0.01$. 
astigmatism less than $1.00 \mathrm{D}$; could have corrected visual acuity of both eyes at 1.0; could master the scientific lenses wear method; had complete clinical data; and cooperated with correction treatment, follow up and regular review.

\section{Exclusion criteria}

Patients were excluded if they had immunological defects, congenital defects, mental diseases, asthma or malignant tumors and needed long-term medication; or were unfit for the correction plans.

\section{Instruments and materials}

Overnight OK contact lenses (Equines II, oxygen permeability 90-110, geometric reverse four-arc surface) applied Corneal Refractive Technology. The slit lamp microscope (YZ5S) was purchased from Topcon, Japan. Corneal topography (TM-4) was purchased from Tomey, Japan. Keratometer (ARK-510A) was purchased from Nidek, Japan. Iolmaster was purchased from Zeiss, Germany. Non-contact tonometer (Canon TX-F) was purchased from Canon, Japan. Corneal endothelial microscope was purchased from Nidek, Japan. VI-Cell

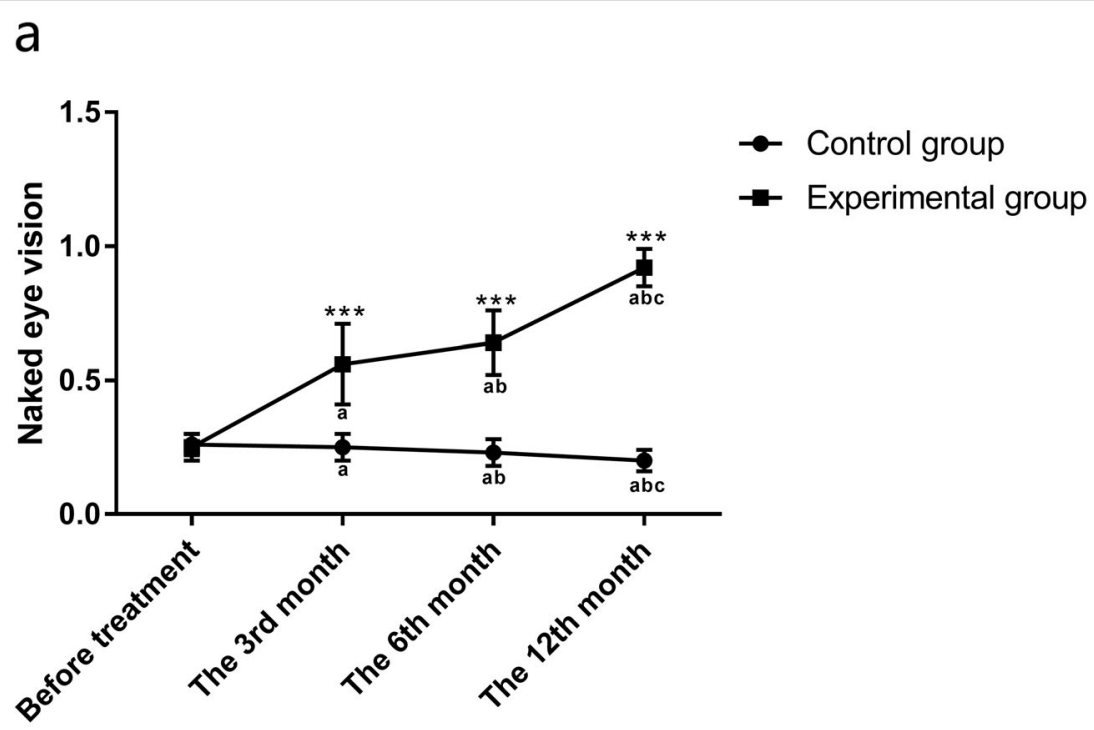

b

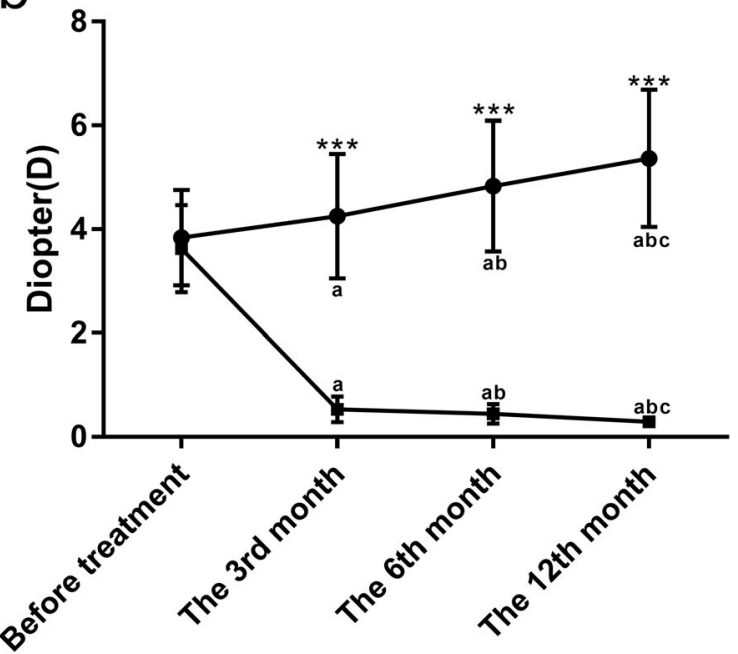

$\rightarrow$ Control group

$\rightarrow$ Experimental group

Fig. 1 Naked-eye vision and diopter in the two groups. a: The naked-eye vision of the control group was significantly lower than that of the experimental group at the $3 \mathrm{rd}$, 6th and 12th months $\left({ }^{* * *} p<0.001\right)$. b: The diopter of the control group was significantly higher than that of the experimental group at the $3 \mathrm{rd}$, 6th and 12 th months $\left({ }^{* * *} p<0.001\right)$. Compared with before treatment, ${ }^{\text {a }} p<0.01$; compared with the 3 rd month, ${ }^{b} p<0.01$; compared with the 6th month, ${ }^{c} p<0.01$ 
Table 5 Changes of corneal curvature (D)

\begin{tabular}{lllll}
\hline Group & $\begin{array}{l}\text { Control group } \\
(n=94)\end{array}$ & $\begin{array}{l}\text { Experimental group } \\
(n=106)\end{array}$ & $\mathrm{t}$ & $p$ \\
\hline Before treatment & $43.40 \pm 1.30$ & $43.25 \pm 1.54$ & 0.747 & 0.456 \\
The 3rd month & $43.45 \pm 1.38$ & $40.28 \pm 1.23^{\mathrm{a}}$ & 17.058 & 0.000 \\
The 6th month & $43.50 \pm 1.29$ & $39.62 \pm 1.12^{\mathrm{ab}}$ & 22.298 & 0.000 \\
The 12th month & $43.52 \pm 1.42$ & $39.38 \pm 1.32^{\mathrm{ab}}$ & 21.268 & 0.000 \\
\hline
\end{tabular}

Compared with before treatment, ${ }^{a} p<0.01$; compared with the 3rd month, ${ }^{b} p<0.01$

XR corneal endothelial cell counter (CEM-530) was purchased from Beckman Coulter, USA.

\section{Methods \\ Optometry}

Visual acuity of patients was tested by a logarithmic visual chart at a distance of $5 \mathrm{~m}$. The uncorrected visual acuity referred to the logarithm of the smallest row that the patient saw (two eyes were tested separately), which was tested starting with pointing the letters from big ones to small ones. A slit lamp was used to examine the patient's eyes from right to left to see if there was any pathological or morphological change. The nerve nipples, macular area, retinal vessels and retina of patients were examined. During the examination, patients should roll their eyeball to ensure that no relevant diseases occurred. The keratometer and corneal topography were used to detect the overall morphology of the patient's cornea so as to evaluate the optical characteristics. The tests were carried out for 3 times repeatedly. The number and regularity of corneal endothelial cells were detected by corneal endothelial cell counter.

\section{Wear method}

The frame glasses in control group were made and wore according to the conventional method, while patients in the experimental group wore the OK lenses for 8-10 h overnight. Eye drop was given after awaking in the morning, and $10 \mathrm{~min}$ later the lenses were removed.

\section{Outcome measures}

\section{Main outcome measures}

Follow-up was performed 3 months, 6 months and 12 months after the start of treatment. The naked-eye

Table 6 Changes of intraocular pressure $(\mathrm{mmHg})$

\begin{tabular}{lllll}
\hline Group & $\begin{array}{l}\text { Control group } \\
(n=94)\end{array}$ & $\begin{array}{l}\text { Experimental group } \\
(n=106)\end{array}$ & $\mathrm{t}$ & $p$ \\
\hline Before treatment & $13.25 \pm 1.48$ & $13.25 \pm 1.62$ & 0.000 & 0.999 \\
The 3rd month & $13.20 \pm 1.62$ & $13.21 \pm 1.48$ & 0.045 & 0.964 \\
The 6th month & $13.21 \pm 1.50$ & $13.20 \pm 1.58$ & 0.046 & 0.963 \\
The 12th month & $13.21 \pm 1.56$ & $13.12 \pm 1.53$ & 0.411 & 0.682 \\
\hline
\end{tabular}

Table 7 Changes of axial length $(\mathrm{mm})$

\begin{tabular}{lllll}
\hline Group & $\begin{array}{l}\text { Control group } \\
(n=94)\end{array}$ & $\begin{array}{l}\text { Experimental group } \\
(n=106)\end{array}$ & $\mathrm{t}$ & $p$ \\
\hline Before treatment & $24.55 \pm 0.33$ & $24.58 \pm 0.41$ & 0.565 & 0.573 \\
The 3rd month & $24.79 \pm 0.32^{\mathrm{a}}$ & $24.71 \pm 0.43$ & 1.477 & 0.141 \\
The 6th month & $24.95 \pm 0.41^{\mathrm{ab}}$ & $24.76 \pm 0.43$ & 3.188 & 0.002 \\
The 12th month & $25.26 \pm 0.45^{\mathrm{abc}}$ & $24.89 \pm 0.45$ & 5.804 & 0.000
\end{tabular}

Compared with before treatment, ${ }^{a} p<0.01$; compared with the 3rd month,

${ }^{\mathrm{b}} p<0.01$; compared with the 6th month, ${ }^{c} p<0.01$

vision, diopter, corneal curvature, intraocular pressure, axial length, endothelial cell count and central corneal thickness were examined.

Secondary outcome measures: Complications during the correction period and corneal fluorescein staining grade of patients were observed. See Table 1 for the grading.

\section{Statistical analysis}

In this study, SPSS 20.0 (Shanghai Cabit, China) was used for statistical analyses. GraphPad Prism 7 (Shenzhen SOFTHEAD, China) was used for output of figures. Measurement data were expressed as mean \pm standard deviation, compared between groups by independent sample t-test, and compared within groups between different time points by paired $\mathrm{t}$-test. The counting data were expressed as percentage (\%), processed by chisquare test. There was a statistical difference when $p<0.05$.

\section{Results}

\section{Analysis of clinical data}

There was no statistical difference in clinical data between the two groups (all $p>0.05$ ), so the two groups were comparable. See Table 2.

\section{Naked-eye vision and diopter}

It was found that the naked-eye vision of patients increased gradually in the experimental group, while decreased gradually in the control group. The naked-eye vision of patients in the experimental group was significantly higher than that in the control group at the 3rd, 6th and 12th months (all $p<0.001$ ). The diopter gradually decreased in the experimental group, while gradually increased in the control group, and was

Table 8 Comparison of difference value in intraocular pressure (mmHg)

\begin{tabular}{lllll}
\hline Group & $\begin{array}{l}\text { Control group } \\
(n=94)\end{array}$ & $\begin{array}{l}\text { Experimental group } \\
(n=106)\end{array}$ & $\mathrm{t}$ & $p$ \\
\hline The 3rd month & $0.05 \pm 0.09$ & $0.04 \pm 0.07$ & 0.869 & 0.386 \\
The 6th month & $0.04 \pm 0.05$ & $0.05 \pm 0.06$ & 1.285 & 0.200 \\
The 12th month & $0.04 \pm 0.05$ & $0.03 \pm 0.04$ & 1.594 & 0.123 \\
\hline
\end{tabular}


significantly lower in the experimental group than in the control group at the 3rd, 6th and 12th months (all $p<0.001$ ). There was a difference between any other time points (within group) in both groups in terms of naked-eye vision and diopter (naked-eye vision: $F=$ 32.098 in the control group, $F=727.005$ in the experimental group, both $p<0.001$; diopter: $\mathrm{F}=29.613$ in the control group, $\mathrm{F}=1344.184$ in the experimental group, both $p<0.001)$. See Table 3, Table 4 and Fig. 1.

\section{Corneal curvature, intraocular pressure and axial length during follow-up period}

It was found that the corneal curvature of the experimental group decreased significantly compared with that of the control group at the 3rd, 6th and 12th months of treatment (all $p<0.001$ ). The differences of intraocular pressure between the two groups were not statistically significant before treatment as well as at the 6th and 12th months of treatment (all $p>0.05$ ). The increases of axial length in the experimental group were significantly less than those in the control group at the 6th and 12th months $(p=0.002$ and $p<0.001)$. The differences of corneal curvature and intraocular pressure in the control group between each time point were not significant (corneal curvature: $\mathrm{F}=0.150, p=0.930$; intraocular pressure $F=0.019, p=0.996)$, but there were differences in axial length between each time point $(F=8.345, p<0.001)$. In the experimental group, there was no difference in intraocular pressure and axial length (intraocular pressure $F=0.130, p=0.942$; axial length: $F=1.393, p=0.244$ ), but there were differences in corneal curvature between each time point $(F=196.545, p<0.001)$. See Tables 5, 6, 7, 8 and Fig. 2.

\section{Changes of endothelial cell count and central corneal thickness}

There was no difference in the endothelial cell count and central corneal thickness as well as their difference values between the two groups (all $p>0.05$ ). Additionally, the differences of endothelial cell count and central corneal thickness within each group between different time points were also not significant (all $p>0.05$ ). See Tables 9, 10, 11, 12 and Fig. 3.

\section{Complications and corneal fluorescein staining}

We found that the incidence of complications in the control group was significantly lower than that in the experimental group $\left(\chi^{2}=4.913, p=0.027\right)$, as shown in Table 13. There was no statistical difference in the grade of corneal fluorescein staining between the two groups $(Z=-0.255, p=0.799)$. See Table 14 .

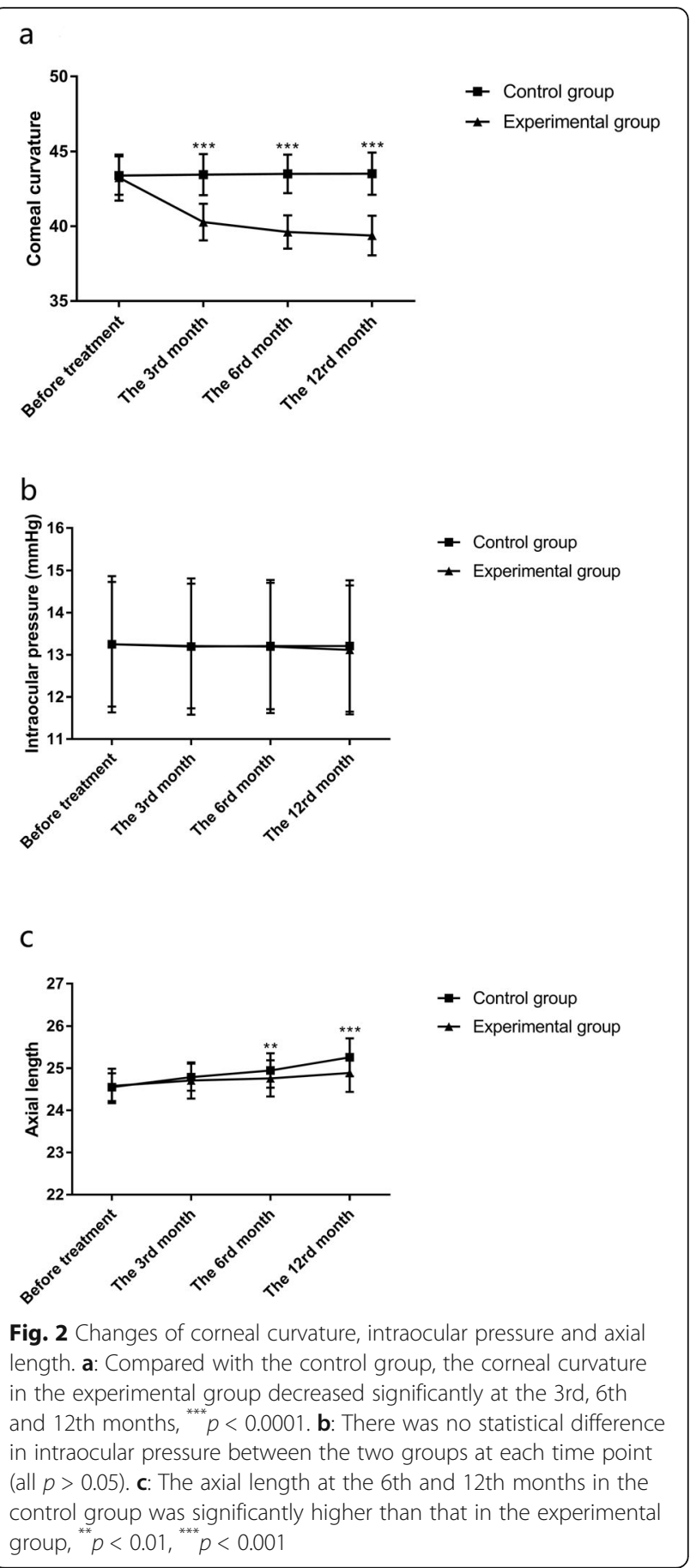

\section{Discussion}

Scholars have suggested that myopia is caused by both genetic and environmental factors, while other studies have shown that long-hour work and reading at short range can also lead to myopia [14-17]. The incidence of myopia among adolescents has increased significantly mainly because their eyeball is in a state of adjustment during long-hour short-range reading. Excessive adjustment causes ciliary muscle to contract continuously, 
Table 9 Changes of endothelial cell count

\begin{tabular}{lllll}
\hline Group & $\begin{array}{l}\text { Control group } \\
(n=94)\end{array}$ & $\begin{array}{l}\text { Experimental group } \\
(n=106)\end{array}$ & $\mathrm{t}$ & $p$ \\
\hline Before treatment & $3345.15 \pm 138.54$ & $3332.72 \pm 142.88$ & 0.624 & 0.533 \\
The 3rd month & $3332.85 \pm 129.68$ & $3328.51 \pm 126.36$ & 0.239 & 0.811 \\
The 6th month & $3329.27 \pm 118.61$ & $3326.39 \pm 113.84$ & 0.175 & 0.862 \\
The 12th month & $3316.17 \pm 106.51$ & $3318.84 \pm 99.74$ & 0.182 & 0.856 \\
\hline
\end{tabular}

eventually leading to function decline and axial myopia. Survey showed that the incidence of myopia among teenagers is positively related to the increase of school work, and the incidence among adolescents in urban areas is significantly higher than that in rural areas [18]. It is urgent for medical staff to find a better way for myopia treatment and prevention.

OK lens is a kind of auxiliary treatment, which differs from ordinary contact lens in terms of design concept, technology and materials. The OK lens adopts a multiarc design, which flattens patients' cornea, reshapes the corneal curvature, improves visual acuity, and avoids the progress of myopia [19]. However, it is not clear whether there is a safety problem when OK lens directly contacts the cornea of patients. Therefore, this study aimed to explore the correction effects of short-term OK lens or ordinary frame glasses wear, and the influence on central corneal thickness and corneal endothelial cells in adolescents with low to moderate myopia to provide a basis for clinicians in terms of correction plans.

Wen's study showed that the naked-eye vision of juvenile myopia patients was obviously improved and the diopter was reduced by wearing OK lenses [20]. In this study, we compared two groups of patients and found that the naked-eye vision in control group (ordinary frame glasses) was significantly lower than that in the experimental group (OK lenses) at each time point, and the diopter in the control group was significantly increased, with significant differences when comparing with that in the experimental group at each time point. It can be indicated that the OK lens significantly improved the visual acuity and diopter in juvenile patients. Wang's 6-month study showed that OK lenses wear had no significant impact on patients' intraocular pressure, and the axial length in patients wearing frame glasses was significantly longer than that in patients wearing

Table 10 Comparison of difference value in endothelial cell count

\begin{tabular}{lllll}
\hline Group & $\begin{array}{l}\text { Control group } \\
(n=94)\end{array}$ & $\begin{array}{l}\text { Experimental group } \\
(n=106)\end{array}$ & $\mathrm{t}$ & $\mathrm{p}$ \\
\hline The 3rd month & $30.08 \pm 25.21$ & $28.54 \pm 21.76$ & 0.460 & 0.646 \\
The 6th month & $34.68 \pm 23.58$ & $30.84 \pm 25.42$ & 1.108 & 0.269 \\
The 12th month & $44.48 \pm 38.54$ & $40.35 \pm 35.84$ & 0.782 & 0.435 \\
\hline
\end{tabular}

Table 11 Changes of central corneal thickness ( $\mu \mathrm{m})$

\begin{tabular}{lllll}
\hline Group & $\begin{array}{l}\text { Control group } \\
(n=94)\end{array}$ & $\begin{array}{l}\text { Experimental group } \\
(n=106)\end{array}$ & $\mathrm{t}$ & $p$ \\
\hline Before treatment & $533.55 \pm 30.51$ & $531.85 \pm 31.05$ & 0.390 & 0.697 \\
The 3rd month & $531.84 \pm 29.18$ & $529.84 \pm 29.58$ & 0.481 & 0.631 \\
The 6th month & $529.18 \pm 28.39$ & $528.11 \pm 28.12$ & 0.267 & 0.790 \\
The 12th month & $528.10 \pm 27.92$ & $527.62 \pm 27.62$ & 0.122 & 0.903 \\
\hline
\end{tabular}

OK lenses [21]. We also detected the changes of corneal curvature, axial length and intraocular pressure in the two groups of patients. We found that the corneal curvature in the control group was higher than that in the experimental group at each time point in the correction process. Although the axial length increased in both groups, it was significantly lower in the experimental group than in the control group at the 6th and 12th month of correction. The intraocular pressure between the two groups had no significant difference during the correction process. Our results indicate that OK lenses can improve the corneal curvature and axial length, and have no obvious influence on the intraocular pressure in myopic patients, which is beneficial to the correction of visual acuity. Study of Zhou et al. found that the corneal curvature of patients wearing OK lenses was significantly lower than that of patients wearing frame glasses, which is consistent with our results [22]. We also examined the corneal thickness and corneal endothelial cells in two groups of patients, and found no difference in the two indexes between the two groups. The results indicate that although the OK lens directly contacts patients' cornea, it has no significant influence on corneal thickness and endothelial cell count. Besides, we found no significant difference in the corneal fluorescein staining grade between the two groups. The incidence of complications in the control group was significantly lower than that in the experimental group. It indicates that patients wearing OK lenses are prone to have complications. But we found that the main cause of complications in the experimental group was the irregular wear method and daily cleaning, and the complications were reduced after giving guidance to the patients.

However, there are still some limitations in this study. Firstly, the follow-up period was not long enough; the mechanism of action has not been explored in depth;

Table 12 Comparison of difference value in central corneal thickness $(\mu \mathrm{m})$

\begin{tabular}{lllll}
\hline Group & $\begin{array}{l}\text { Control group } \\
(n=94)\end{array}$ & $\begin{array}{l}\text { Experimental group } \\
(n=106)\end{array}$ & $\mathrm{t}$ & $p$ \\
\hline The 3rd month & $4.92 \pm 2.35$ & $4.21 \pm 3.12$ & 1.830 & 0.070 \\
The 6th month & $5.34 \pm 2.35$ & $4.84 \pm 2.12$ & 1.572 & 0.118 \\
The 12th month & $6.36 \pm 3.84$ & $6.64 \pm 4.15$ & 0.496 & 0.621 \\
\hline
\end{tabular}


a
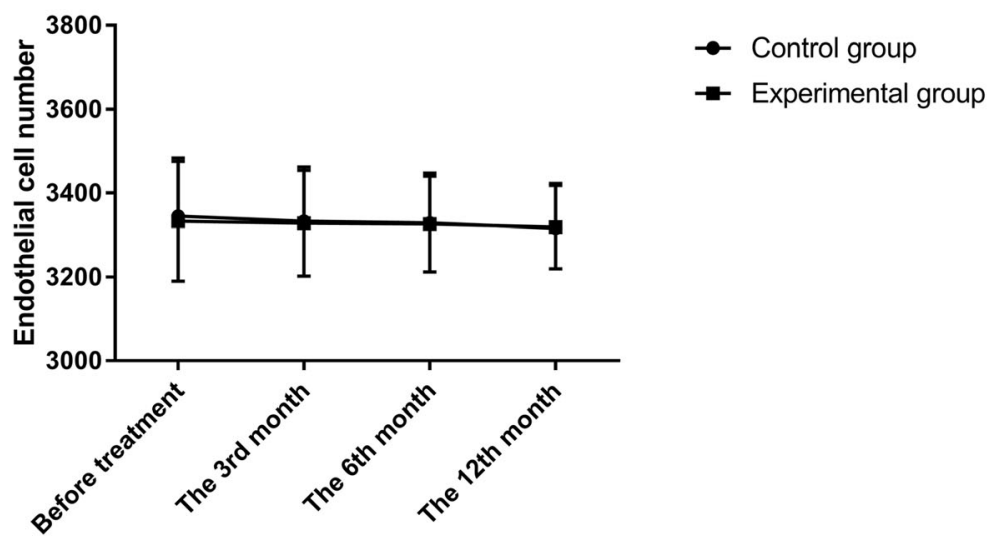

b

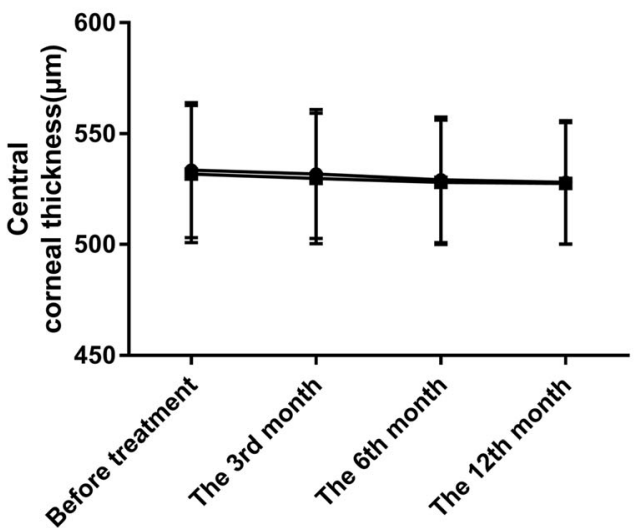

Control group

- Experimental group

Fig. 3 Changes of endothelial cell count and central corneal thickness. a: There was no statistical difference in endothelial cell count in both groups between each time point (all $p>0.05$ ). $\mathbf{b}$ : There was no statistical difference in central corneal thickness in both groups between each time point (all $p>0.05$ )

indexes such as changes of tear film and peripheral corneal thickness were not analyzed. Secondly, it is not clear whether reading time and/or screen time had any impact on the study results for we failed to record the relevant data. Lastly, we used both eyes from each participant, which may cause confounding in the study results. Therefore, we will carry out further studies with larger sample size, longer follow-up, more adequate detection indexes to explore the mechanism and to verify

Table 13 Comparison of complications ( $n, \%$ )

\begin{tabular}{lllllll}
\hline Group & $\begin{array}{l}\text { Foreign } \\
\text { body } \\
\text { sensation }\end{array}$ & $\begin{array}{l}\text { Visual } \\
\text { abnormality }\end{array}$ & $\begin{array}{l}\text { Aseptic } \\
\text { infiltration }\end{array}$ & Keratitis & $\begin{array}{l}\text { Corneal } \\
\text { virus } \\
\text { infection }\end{array}$ & Total \\
\hline $\begin{array}{l}\text { Control group } \\
(n=47)\end{array}$ & $0(0.00)$ & $2(4.26)$ & $0(0.00)$ & $1(2.13)$ & $1(2.13)$ & $\begin{array}{l}4 \\
(8.51)\end{array}$ \\
$\begin{array}{l}\text { Experimental } \\
\text { group } \\
(n=53)\end{array}$ & $2(3.77)$ & $3(5.66)$ & $1(1.89)$ & $3(5.66)$ & $2(3.77)$ & 11 \\
& & & & & & $(20.75)$ \\
\hline
\end{tabular}

our results. In recent years, there is a kind of soft eye contact lenses in the market. Report showed the safety and convenience of soft lenses as compared with the rigid lenses [23]. But at present there are no reports about its long-term safety. Therefore, we also hope to delve into this aspect in future studies, so as to provide reference for clinical treatment.

\section{Conclusions}

In summary, short-term OK lens wear can effectively improve the naked-eye vision in adolescents with low to

Table 14 Grade of corneal fluorescein staining

\begin{tabular}{llllll}
\hline Group & 0 & $\mathrm{I}$ & $\mathrm{I}$ & $\mathrm{III}$ & $\mathrm{IV}$ \\
\hline $\begin{array}{l}\text { Control group } \\
(n=47)\end{array}$ & $40(85.11)$ & $4(8.51)$ & $1(2.13)$ & $1(2.13)$ & $1(2.13)$ \\
$\begin{array}{l}\text { Experimental group } \\
(n=53)\end{array}$ & $44(83.02)$ & $5(9.43)$ & $3(5.66)$ & $1(1.89)$ & $0(0.00)$ \\
\hline
\end{tabular}


moderate myopia without significant impact on the central corneal thickness and corneal endothelial cells. It is a relatively safe method to correct myopia. And it is necessary to master the correct and scientific optometry as well as methods for cleaning and preservation to better play its role.

\section{Abbreviation \\ OK: orthokeratology}

\section{Acknowledgements}

Not applicable.

\section{Authors' contributions}

SY contributed to study design and statistical analysis; SZ contributed to literature research, clinical studies and manuscript preparation; $Y J$ contributed to manuscript review and data acquisition; LL was the guarantor of integrity of the entire study and contributed to study concepts, experimental studies and data analysis;: The final version of the manuscript has been read and approved by all authors, and each author believes that the manuscript represents honest work.

\section{Funding}

No funding was received.

\section{Availability of data and materials}

The analysed datasets generated during the study are available from the corresponding author on reasonable request.

\section{Ethics approval and consent to participate}

This study was approved by the Medical Ethics Committee of Tianjin Eye Hospital. Witten informed consent form was obtained from all patients and their guardians.

\section{Consent for publication}

Not applicable.

\section{Competing interests}

The authors declare that they have no competing interests.

Received: 14 May 2019 Accepted: 21 October 2019

Published online: 28 November 2019

\section{References}

1. Dolgin E. The myopia boom. Nature. 2015;519(7543):276-8.

2. Williams KM, Venturini C, Yonova EH, Hysi PG, Plomin R, Hammond CJ. Evidence for shared genetic factors between myopia and intelligence in the twins early development study (TEDS). Minn Hist. 2015:44(5):185-8.

3. Hou W, Norton TT, Hyman L, Gwiazda J. Axial elongation in myopic children and its association with myopia progression in the correction of myopia evaluation trial. Eye \& contact lens. 2018;44(4):248-59.

4. Holden BA, Fricke TR, Wilson DA, et al. Global prevalence of myopia and high myopia and temporal trends from 2000 through 2050. Ophthalmology. 2016;123(5):1036-42.

5. Woo R, Akil H, Koulisis N. Olmos de Koo LC, tan JCH. Sustained resolution of macular Retinoschisis after trabeculectomy in a patient with progressive Glaucoma. J Glaucoma. 2017:26(6):e180-6.

6. Kang MT, Li SM, Peng X, et al. Chinese eye exercises and myopia development in school age children: a nested case-control study. Sci Rep. 2016;6:28531.

7. Scheiman M, Gwiazda J, Zhang Q, et al. Longitudinal changes in corneal curvature and its relationship to axial length in the correction of myopia evaluation trial (COMET) cohort. J Opt. 2016;9(1):13-21.

8. Morjaria P. Murali K, Evans J, Gilbert C. Spectacle wearing in children randomised to ready-made or custom spectacles, and potential cost savings to programmes: study protocol for a randomised controlled trial. Trials. 2016;17:36.

9. Dugel PU, Hillenkamp J, Sivaprasad S, et al. Baseline visual acuity strongly predicts visual acuity gain in patients with diabetic macular edema following anti-vascular endothelial growth factor treatment across trials. Clinical ophthalmology (Auckland, NZ). 2016:10:1103-1110.

10. Nelson BA, Gunton KB, Lasker JN, Nelson LB, Drohan LA. The psychosocial aspects of strabismus in teenagers and adults and the impact of surgical correction. Journal of American Association for Pediatric Ophthalmology and Strabismus. 2008;12(1):72-6.

11. Swarbrick HA, Alharbi A, Watt K, Lum E, Kang P. Myopia control during orthokeratology lens wear in children using a novel study design. Ophthalmology. 2015;122(3):620-30.

12. Han $X, X u$ D, Ge W, Wang Z, Li X, Liu W. A comparison of the effects of Orthokeratology Lens, Medcall Lens, and ordinary frame glasses on the accommodative response in myopic children. Eye \& contact lens. 2018;44(4): 268-71

13. Bron AJ, Evans VE, Smith JA. Grading of corneal and conjunctival staining in the context of other dry eye tests. Cornea. 2003;22(7):640-50.

14. Goldschmidt E, Jacobsen N. Genetic and environmental effects on myopia development and progression. Eye (London, England). 2014;28(2):126-133.

15. Guo L, Yang J, Mai J, et al. Prevalence and associated factors of myopia among primary and middle school-aged students: a school-based study in Guangzhou. Eye (London, England). 2016;30(6):796-804.

16. Recko M, Stahl ED. Childhood myopia: epidemiology, risk factors, and prevention. Mo Med. 2015:112(2):116-21.

17. Rudnicka AR, Kapetanakis W, Wathern AK, et al. Global variations and time trends in the prevalence of childhood myopia, a systematic review and quantitative meta-analysis: implications for aetiology and early prevention. Br J Ophthalmol. 2016;100(7):882-90.

18. Epstein D. The correlation between the amplitude of accommodation and low-luminance myopia. Acta Ophthalmol. 1984;62(6):955-60.

19. Zhang H, Zhao F, Hutchinson DS, et al. Conjunctival microbiome changes associated with soft contact Lens and Orthokeratology Lens wearing. Invest Ophthalmol Vis Sci. 2017;58(1):128-36

20. Wen D, Huang J, Chen H, et al. Efficacy and acceptability of Orthokeratology for slowing myopic progression in children: a systematic review and metaanalysis. J Ophthalmol. 2015;2015:360806.

21. Wang $X$, Zhang L, Qiu $Y$, et al. Influence of orthokeratology lens on vision,intraocular pressure and biometric measurement parameters of adolescent myopia. Chongqing Medicine 2017.

22. Zhou ZX, Xu SS, Yi SP. Hospital NT. Medicine SO: University S. Clinical effect of orthokeratology for juvenile with myopia astigmatism and its effects on corneal endothelial cells. International Eye Science; 2016

23. Ramakrishnan R, Kadu AD, Naik A. Corneal changes in soft contact lens wearers. Journal of Clinical Ophthalmology and Research. 2016:4(1):40.

\section{Publisher's Note}

Springer Nature remains neutral with regard to jurisdictional claims in published maps and institutional affiliations.

Ready to submit your research? Choose BMC and benefit from:

- fast, convenient online submission

- thorough peer review by experienced researchers in your field

- rapid publication on acceptance

- support for research data, including large and complex data types

- gold Open Access which fosters wider collaboration and increased citations

- maximum visibility for your research: over $100 \mathrm{M}$ website views per year

At BMC, research is always in progress.

Learn more biomedcentral.com/submissions 